\title{
Two- and Three-Dimensional Fast Intrawall Imaging with Microwave Tomographic Algorithm
}

\author{
Wenji Zhang, Ahmad Hoorfar (D), and Christopher Thajudeen \\ Antenna Research Laboratory, Department of Electrical and Computer Engineer, Villanova University, Villanova, PA 19085, USA \\ Correspondence should be addressed to Ahmad Hoorfar; ahoorfar@villanova.edu
}

Received 30 May 2017; Revised 15 October 2017; Accepted 7 November 2017; Published 9 January 2018

Academic Editor: Ana Alejos

Copyright (c) 2018 Wenji Zhang et al. This is an open access article distributed under the Creative Commons Attribution License, which permits unrestricted use, distribution, and reproduction in any medium, provided the original work is properly cited.

\begin{abstract}
A fast and efficient microwave tomographic algorithm is proposed for 2-D and 3-D real-time intrawall imaging. The exploding reflection model is utilized to simplify the imaging formulation, and the half-space Green's function is expanded in the spectral domain to facilitate the easy implementation of the imaging algorithm with the fast Fourier transform (FFT) and inverse fast Fourier transform (IFFT). The linearization of the inversion scheme and employment of FFT/IFFT in the imaging formula make the algorithm suitable for various applications pertaining to the inspection of a large probed region and allow real-time processing. Representative numerical and experimental results are presented to show the effectiveness and efficiency of the proposed algorithm for real-time intrawall characterization.
\end{abstract}

\section{Introduction}

Ground penetrating radar (GPR) provides unique noninvasive means for retrieving information on the internal construction of the building walls. The ability to determine fidelity information of the wall, such as hidden wires and tubes, cracks, and voids, and/or the existence of reinforcements is beneficial for both military and civilian applications, that is, building safety and durability assessment, bridge/dam defect characterization, and through-the-wall radar imaging (TWRI) [1-8].

The detection and imaging of concealed targets inside a visually opaque building wall can be addressed by means of the inverse scattering technique. In the past decade, various linear and nonlinear inverse scattering algorithms have been developed for nondestructive testing of abnormalities in concrete structures. In [9], a nonlinear inversion scheme based on the Gauss-Newton method is presented for the quantitative imaging of reinforcement bars in the concrete. The nonlinear inverse scattering algorithm is capable of quantitative reconstruction of the targets with super-resolution. However, it is based on an optimization scheme and thus is CPU intensive and very time-consuming. To accelerate the imaging speed, linear inverse scattering algorithms based on Born or Rytov approximations are proposed for radar imaging [10-13]. Linear inversion schemes ignore multiple scattering effects and thus are only capable of reconstruction of weak scatterers. For strong scattering objects, it yields a qualitative reconstruction; that is, only the geometric properties, such as location and shape, are reconstructed [12, 13]. In [14], a qualitative imaging approach using the half-space Green's function in combination with truncated singular value decomposition is proposed for reinforced concrete diagnostics. The multiple signal classification algorithm and linear sampling method are applied to nondestructive imaging of concrete structures in [15-17]. Backprojection algorithms based on ray tracing technique taking into account the wave propagation velocity in the concrete have been widely used for intrawall nondestructive inspection in $[18,19]$.

Although successful imaging results can be achieved with the aforementioned algorithms, these algorithms mainly deal with 2-D intrawall imaging, which only reconstruct the cross sections of the concealed objects in the wall. In [20], 3-D imaging is applied to damage the assessment of the concrete structure using the delay-and-sum beamforming algorithm. A strategy for 3-D imaging through the fusion of 2-D images for concrete and pavement imaging is presented in [21]. 3-D imaging provides valuable information about the target's 
extent in length, height, and width for enhanced target characterization and feature extraction. Most existing 3-D imaging techniques are computationally intensive and are usually processed off-line. For many applications, it is desirable that the intrawall imaging algorithms are computationally efficient such that the localization of the concealed objects inside the wall can be efficiently determined, preferably within a few seconds for 2-D imaging and a few minutes for 3-D imaging with a portable computer.

In this paper, a fast and efficient microwave tomographic algorithm is proposed for 2-D and 3-D real-time intrawall imaging. The imaging algorithm is based on the first-order Born approximation and exploits the half-space Green's function. The exploding reflection model is employed, and then, the Green's function is expanded in the spectrum domain to facilitate the implementation of the imaging algorithm with the fast Fourier transform (FFT) and inverse fast Fourier transform (IFFT). The linearization of the inversion scheme and employment of FFT/IFFT in the imaging formula make the imaging algorithm suitable for various applications pertaining to the inspection of large probed domain and allow near real-time processing.

\section{Problem Formulation}

2.1. Three-Dimensional Intrawall Imaging. Consider a typical scenario for 3-D intrawall inspection using monostatic synthetic aperture radar (SAR), as shown in Figure 1. The transmitter transmits an ultra-wideband (UWB) electromagnetic signal and collects the reflected signal over a rectangular planar aperture that may have one or more targets within its field of view. The reflected signal is recorded coherently by the receiver, digitized, and stored in the computer. Then, the region between the front and back walls can be efficiently reconstructed to form a focused image of the targets inside the wall using a subsurface imaging algorithm.

As shown in Figure 1, the radar operates in region I, which is the free-air in front of the wall. The targets of interest are embedded in region II, which is the building wall whose dielectric constant and thickness are denoted as $\varepsilon_{b}$ and $d$, respectively. As opposed to TWRI, which aims at imaging of targets behind the wall (region III) [5-8], intrawall imaging is interested in the imaging of targets inside the wall (region II). The radar is located at $\mathbf{r}_{R}=\widehat{x} x_{R}+$ $\hat{y} y_{R}+\widehat{z} z_{R}$ and operates over the frequency range from $f_{\min }$ to $f_{\max }$. We assume the investigated domain is a cuboid region denoted as $D_{\text {inv }}=\left[-x_{\min }, x_{\max }\right] \times\left[-y_{\min }, y_{\max }\right] \times\left[-z_{\min }, z_{\max }\right]$. The complex permittivity inside $D_{\text {inv }}$ is defined as

$$
\varepsilon(\mathbf{r})= \begin{cases}\tilde{\varepsilon}(\mathbf{r}), & \mathbf{r} \in \text { target } \\ \varepsilon_{b}, & \text { otherwise }\end{cases}
$$

where $\mathbf{r}=\hat{x} x+\hat{y} y+\widehat{z} z$ is the position vector of the target.

The scattered electric field observed at the receiver location can be expressed as

$$
E_{s}\left(\mathbf{r}_{R}, k\right)=k^{2} \int G\left(\mathbf{r}_{R}, \mathbf{r}, k\right) E_{\mathrm{t}}\left(\mathbf{r}, \mathbf{r}_{R}, k\right) \mathrm{O}(\mathbf{r}, k) d \mathbf{r},
$$

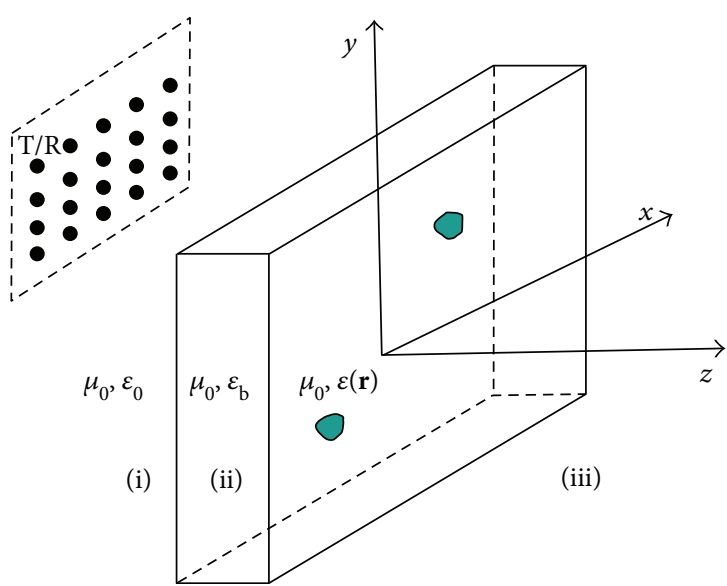

FIgURE 1: Configuration of 3-D intra-wall imaging.

where $k$ is the wavenumber in the free space, $E_{\mathrm{t}}\left(\mathbf{r}, \mathbf{r}_{R}, k\right)$ is the total electrical field inside the target, and $\mathrm{O}(\mathbf{r}, k)$ is the contrast function defined as

$$
\mathrm{O}(\mathbf{r}, k)=\tilde{\varepsilon}(\mathbf{r})-\varepsilon_{b},
$$

where $\tilde{\varepsilon}(\mathbf{r})$ is the relative complex permittivity profile of the target and $G\left(\mathbf{r}_{R}, \mathbf{r}, k\right)$ is the dyadic half-space Green's function which can be expressed in spectrum domain as $[12,22]$

$$
\begin{aligned}
G\left(\mathbf{r}_{R}, \mathbf{r}, k\right)=\frac{j}{8 \pi^{2}} \int_{-\infty}^{\infty} \int_{-\infty}^{\infty} & d k_{x} d k_{y} F\left(k_{x}, k_{y}, k\right) \\
& \cdot \exp \left(j k_{x}\left(x_{R}-x\right)+j k_{y}\left(y_{R}-y\right)\right. \\
& \left.+j k_{2 z} z_{R}-j k_{1 z} z\right)
\end{aligned}
$$

where

$$
\begin{aligned}
F= & \frac{2}{\left(k_{0 z}+k_{1 z}\right)\left(k_{x}^{2}+k_{y}^{2}+k_{0 z} k_{1 z}\right)} \\
& \cdot\left[\widehat{x}\left(\left(k_{x}^{2}+k_{0 z} k_{1 z}\right) \widehat{x}-k_{x} k_{y} \widehat{y}-k_{y} k_{0 z} \widehat{z}\right)\right. \\
& +\widehat{y}\left(-k_{x} k_{y} \widehat{x}+\left(k_{y}^{2}+k_{0 z} k_{1 z}\right) \widehat{y}-k_{x} k_{0 z} \widehat{z}\right) \\
& \left.+\widehat{z}\left(-k_{y} k_{1 z} \widehat{x}-k_{x} k_{1 z} \hat{y}+\left(k_{x}^{2}+k_{y}^{2}\right) \widehat{z}\right)\right] .
\end{aligned}
$$

As the total electrical field inside the target is also a function of the contrast function, (2) is a nonlinear equation. To linearize the inversion scheme, the first-order Born approximation can be used, which assumes that the total field inside the target is approximated by the incident field [23]. Then, the mathematical relationship between the scattered field and the contrast function can be written as

$$
E_{s}\left(\mathbf{r}_{R}, k\right)=k^{2} \int G\left(\mathbf{r}_{R}, \mathbf{r}, k\right) E_{\text {inc }}\left(\mathbf{r}, \mathbf{r}_{R}, k\right) \mathrm{O}(\mathbf{r}, k) d \mathbf{r}
$$




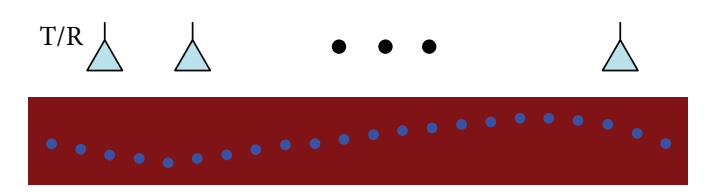

(a)

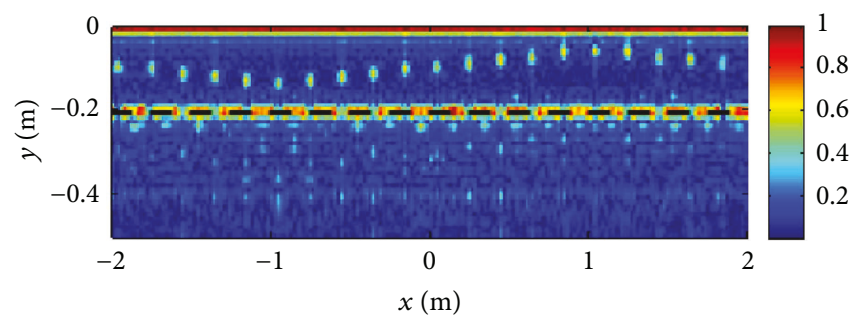

(c)

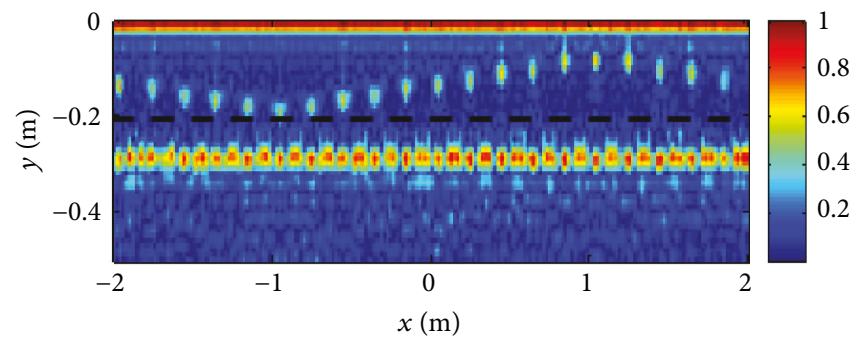

(e)

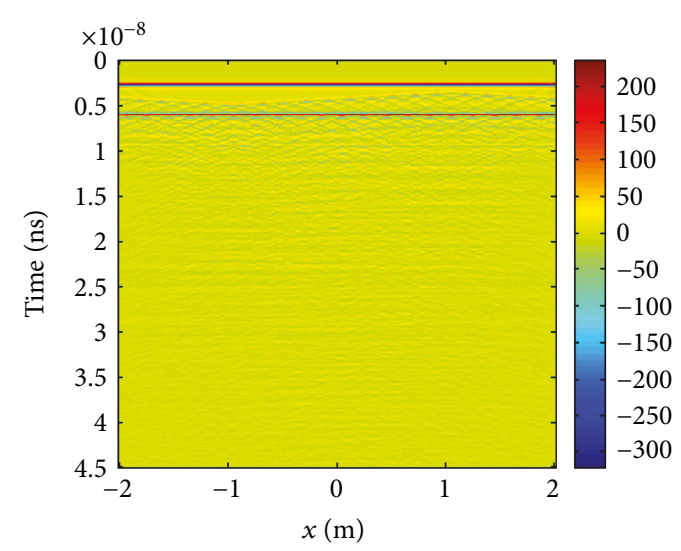

(b)

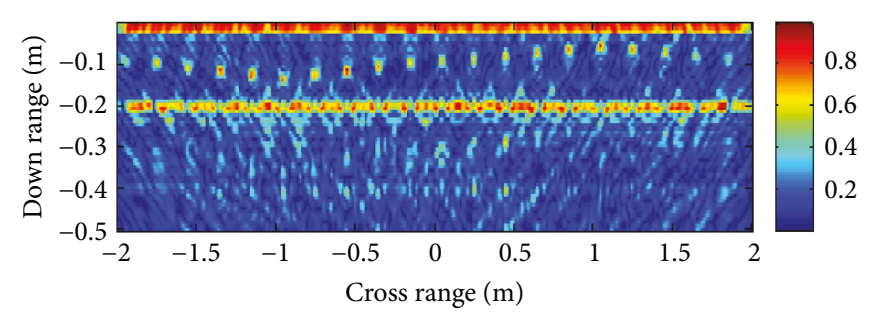

(d)

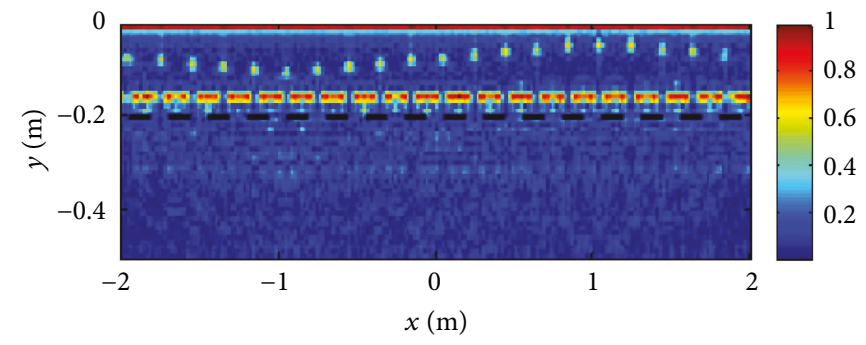

(f)

FIGURE 2: Imaging of a reinforced wall. (a) Geometrical distribution of the steel bars inside the wall. (b) Time domain raw data. (c) Imaging result using the proposed algorithm, correct wall parameter, $\varepsilon_{b}=6$. (d) Imaging result using the ray tracing-based back-projection algorithm, correct wall parameter, $\varepsilon_{b}=6$. (e) Imaging result using the proposed algorithm, underestimated wall parameter, $\varepsilon_{b}=3$. (f) Imaging result using the proposed algorithm, overestimated wall parameter, $\varepsilon_{b}=10$.

where the incident field is given by

$$
E_{\text {inc }}\left(\mathbf{r}, \mathbf{r}_{R}, k\right)=j \omega \mu_{0} G\left(\mathbf{r}, \mathbf{r}_{R}, k\right) \widehat{\alpha}_{T}
$$

wherein $\widehat{\alpha}_{T}$ is the antenna polarization direction.

Substituting (7) into (6) gives

$$
E_{s}\left(\mathbf{r}_{R}, k\right)=j \eta_{0} k^{3} \int\left(G\left(\mathbf{r}_{R}, \mathbf{r}, k\right) \widehat{\alpha}_{T}\right)^{2} \mathrm{O}(\mathbf{r}, k) d \mathbf{r},
$$

where $\eta_{0}$ is the free space wave impedance and the reciprocity relationship $G\left(\mathbf{r}_{R}, \mathbf{r}, k\right)=G\left(\mathbf{r}, \mathbf{r}_{R}, k\right)$ has been used in the above equation.

For simplicity, (8) can be expressed in the following operator form:

$$
E_{s}\left(\mathbf{r}_{R}, k\right)=L(\mathrm{O}(\mathbf{r}))
$$

where the operator $L$ maps the contrast function from the object space to the data space,

$$
L(\cdot) \triangleq j \eta_{0} k^{3} \int_{D_{\mathrm{inv}}}\left(G\left(\mathbf{r}_{R}, \mathbf{r}, k\right) \widehat{\alpha}_{T}\right)^{2}(\cdot) d \mathbf{r}
$$

Then the image can be formulated as

$$
I(\mathbf{r})=L^{a}\left(E_{s}\left(\mathbf{r}_{R}, k\right)\right),
$$

where $L^{a}$ is the adjoint operator of $L$ and maps the scattered field from the data space to the target space $[24,25]$,

$$
L^{a}(\cdot) \triangleq-j \eta_{0} \int_{k_{\min }}^{k_{\max }} k^{3} d k \int\left[\left(G\left(\mathbf{r}_{R}, \mathbf{r}, k\right) \widehat{\alpha}_{T}\right)^{2}\right]^{*}(\cdot) d \mathbf{r}_{R},
$$

where the subscript ${ }^{*}$ is the complex conjugation. From the concept of time reversal imaging, the complex conjugation in the frequency domain is equivalent to the time reversal 


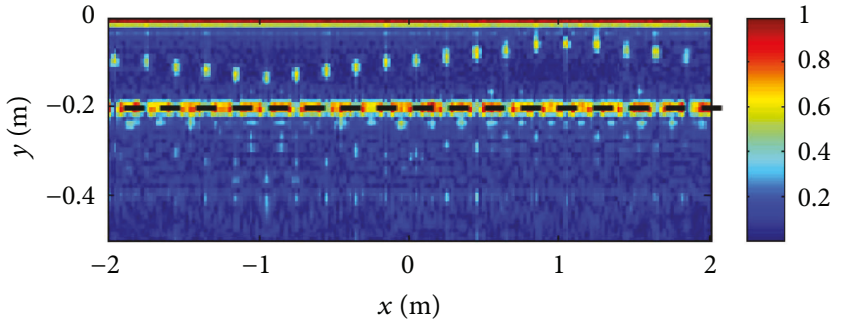

(a)

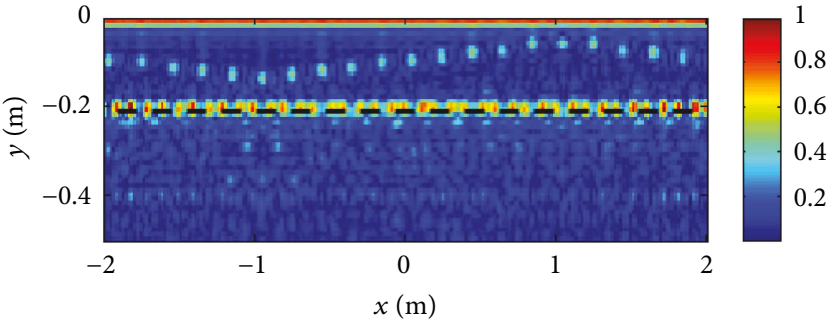

(b)

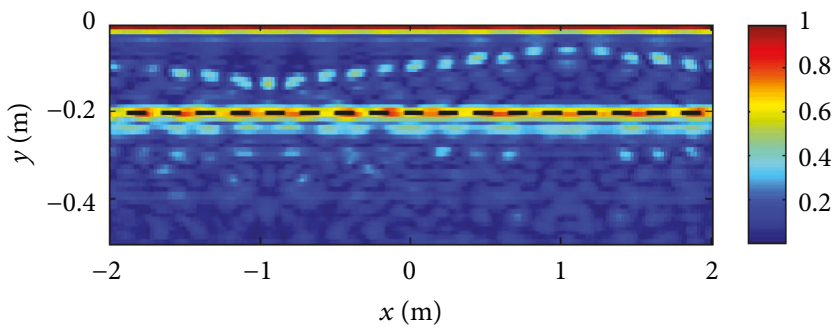

(c)

FIgURE 3: Imaging results with different step sizes. (a) Step size $=2.5 \mathrm{~cm}$. (b) Step size $=5 \mathrm{~cm}$. (c) Step size $=10 \mathrm{~cm}$.

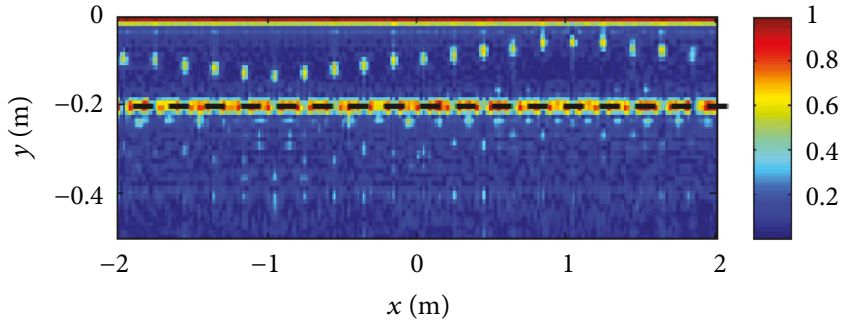

(a)

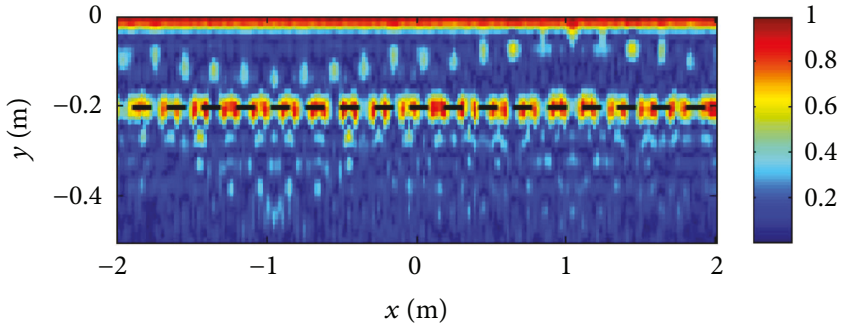

(b)

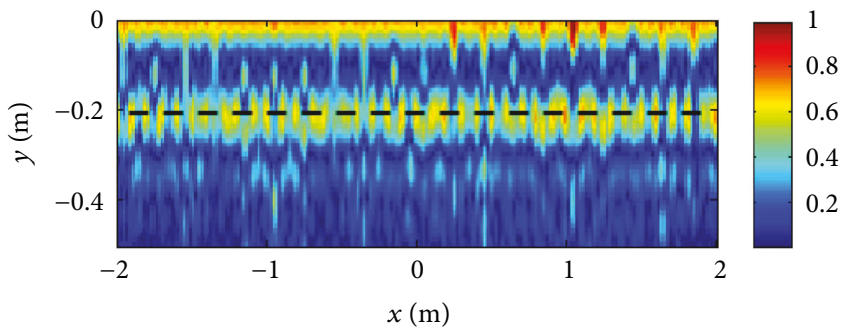

(c)

FIGURE 4: Imaging results with different signal bandwidths. (a) BW=2.4 GHz (Freq $=0.8-3.2 \mathrm{GHz}$ ). (b) BW=1.2 GHz (Freq $=1.4-2.6 \mathrm{GHz}$ ). (c) $\mathrm{BW}=0.6 \mathrm{GHz}($ Freq $=1.7-2.3 \mathrm{GHz})$.

in the time domain. The back propagation of the timereversed field will focus on the target.

It is found from (12) that an efficient evaluation of the subsurface Green's function is critical to the imaging. However, the exact calculation of the dyadic Green's function requires the evaluation of the Sommerfeld integral, which is generally complicated and computationally expensive. To simplify and accelerate the imaging speed, the assumption of exploding reflection model is used $[24,26]$,

$$
\left(G\left(\mathbf{r}_{R}, \mathbf{r}, k\right) \widehat{\alpha}_{T}\right)^{2}=G\left(\mathbf{r}_{R}, \mathbf{r}, 2 k\right) \widehat{\alpha}_{T},
$$

where the squared term, representing the two-way propagation, is replaced by a one-way propagation with half the propagation speed. Then (12) can be further written as

$$
L^{a}(\cdot) \triangleq-j \eta_{0} \int_{k_{\min }}^{k_{\max }} k^{3} d k \int\left[G\left(\mathbf{r}_{R}, \mathbf{r}, 2 k\right) \widehat{\alpha}_{T}\right]^{*}(\cdot) d \mathbf{r}_{R}
$$

It is noticed that the radar system both transmits and receives in the $y$ direction, $\widehat{\alpha}_{T}=\widehat{y}$. By substituting the $3-\mathrm{D}$ spectrum form of the half-space Green's function into (14), the image can be reconstructed as 


$$
\begin{aligned}
I(\mathbf{r})= & L^{a}\left(E_{s}\left(\mathbf{r}_{R}, k\right)\right) \\
=\frac{-\eta_{0}}{8 \pi^{2}} \int_{k_{\min }}^{k_{\max }} k^{3} d k \iint & d k_{x} d k_{y} \tilde{E}_{s}\left(k_{x}, k_{y}, k\right) P\left(k_{x}, k_{y}, k\right) \\
\cdot & \exp \left(j k_{1 z}^{*} z-j k_{2 z}^{*} z_{R}\right) \exp \left(j k_{x} x+j k_{y} y\right),
\end{aligned}
$$

where

$$
\begin{aligned}
P\left(k_{x}, k_{y}, k\right) & =\frac{2\left(k_{y}^{2}+k_{1 z}^{*} k_{2 z}^{*}\right)}{\left(k_{1 z}^{*}+k_{2 z}^{*}\right)\left(k_{x}^{2}+k_{y}^{2}+k_{1 z}^{*} k_{2 z}^{*}\right)}, \\
k_{1 z} & =\sqrt{4 k^{2}-k_{x}^{2}-k_{y}^{2}}, \\
k_{2 z} & =\sqrt{4 k_{b}^{2}-k_{x}^{2}-k_{y}^{2}},
\end{aligned}
$$

and where $k$ and $k_{b}$ are the wavenumbers in the free-space and, in the wall, $\tilde{E}_{s}\left(k_{x}, k_{y}, k\right)$ is the 2-D FFT of $E_{s}\left(\mathbf{r}_{R}, k\right)$ with respect to $x_{R}$ and $y_{R}$,

$$
\tilde{E}_{s}\left(k_{x}, k_{y}, k\right)=\int E_{s}\left(\mathbf{r}_{R}, k\right) \exp \left(-j k_{x} x_{R}-j k_{y} y_{R}\right) d x_{R} d y_{R}
$$

The 3-D intrawall imaging formulation in (15) can now be efficiently implemented in the following steps: (1) perform 2-D FFT to compute the spatial Fourier transform of the scattered field $\tilde{E}_{s}\left(k_{x}, k_{y}, k\right) ;(2)$ multiply $\tilde{E}_{s}\left(k_{x}, k_{y}, k\right)$ with all the functions inside the inner integral except the exponential term $\exp \left(j k_{x} x+j k_{y} y\right)$; and (3) at each down range pixel $z$ applies 2-D IFFT to evaluate the inner integral and then sums over all the frequencies to calculate the outer integral.

2.2. Two-Dimensional Intrawall Imaging. The above derivation of a 3-D imaging algorithm can be simply reduced to 2-D intrawall imaging by replacing the half-space dyadic Green's function with 2-D half-space Green's function [22]

$G\left(\mathbf{r}_{R}, \mathbf{r}, k\right)=\frac{j}{4 \pi} \int_{-\infty}^{\infty} d k_{x} \frac{2}{k_{1 z}+k_{2 z}} \exp \left(\begin{array}{l}j k_{x}\left(x_{R}-x\right) \\ +j k_{2 z} z_{R}-j k_{1 z} z\end{array}\right)$.

Substitute the 2-D half-space Green's function into (14); the 2-D imaging formula can be derived as

$$
\begin{aligned}
& I(\mathbf{r})= L^{a}\left(E_{s}\left(\mathbf{r}_{R}, k\right)\right) \\
&=\frac{-\eta_{0}}{4 \pi} \int_{k_{\min }}^{k_{\max }} k^{3} d k \int d k_{x} \tilde{E}_{s}\left(k_{x}, k\right) \cdot P\left(k_{x}, k\right) \\
& \cdot \exp \left(j k_{1 z}^{*} z-j k_{2 z}^{*} z_{R}\right) \exp \left(j k_{x} x\right),
\end{aligned}
$$

where $P\left(k_{x}, k\right)=2 /\left(k_{1 z}^{*}+k_{2 z}^{*}\right), k_{1 z}=\sqrt{4 k^{2}-k_{x}^{2}}, k_{2 z}=\sqrt{4 k_{b}^{2}-k_{x}^{2}}$, and $\tilde{E}_{s}\left(k_{x}, k\right)$ are the FFT of $E_{s}\left(\mathbf{r}_{R}, k\right)$ with respect to $x_{R}$ :

$$
\tilde{E}_{s}\left(k_{x}, k\right)=\int E_{s}\left(\mathbf{r}_{R}, k\right) \exp \left(-j k_{x} x_{R}\right) d x_{R} .
$$

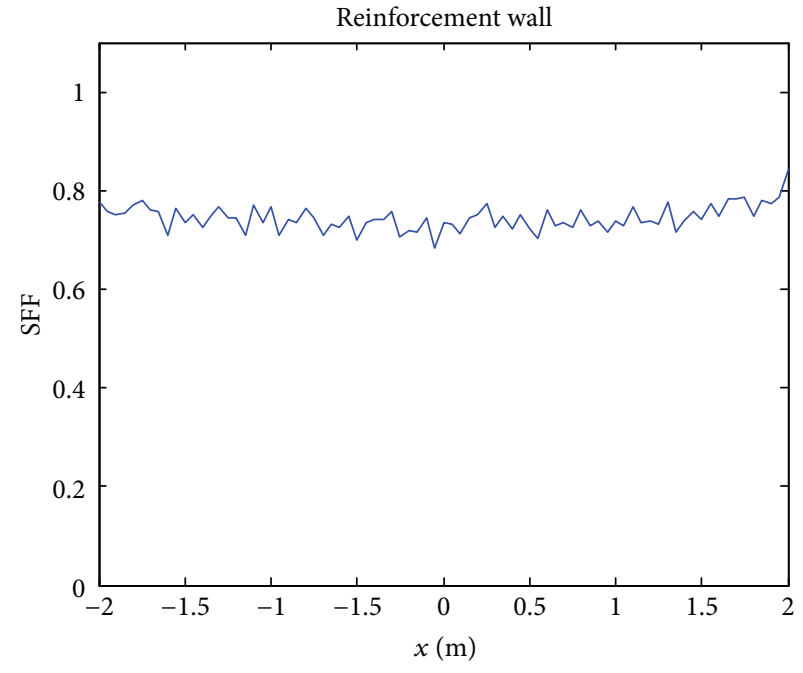

FIGURE 5: SFF of the reinforced wall versus antenna location.

\section{Numerical Results}

Representative numerical examples are presented in this section to verify and show the efficiency of the proposed algorithms for 2-D and 3-D intrawall imaging. In all the following examples, the imaging is performed on a four-core P4 $2.66 \mathrm{GHz}$ desktop computer with $16 \mathrm{~GB}$ of RAM.

In the first example, we present the 2-D imaging of a reinforced wall with a sinusoidal distribution of steel bars inside the wall as shown in Figure 2(a). The diameter of each steel bar is $1 \mathrm{~cm}$ with a spacing of $20 \mathrm{~cm}$ in the horizontal direction. The distribution of the steel bars is an approximate sinusoid function. The received reflected signal was simulated using a full wave electromagnetic solver based on the finite difference time domain (FDTD) method. The monostatic radar measures the return signal at a standoff distance of $0.3 \mathrm{~m}$ over a synthetic aperture extending from $-2 \mathrm{~m}$ to $2 \mathrm{~m}$ with a step of $0.025 \mathrm{~m}$. The dielectric constant, conductivity, and thickness of the wall are $\varepsilon_{b}=6, \sigma=0.01 \mathrm{~S} / \mathrm{m}$, and $d=0.2 \mathrm{~m}$, respectively. The operating frequency is $0.8 \mathrm{GHz}$ to $3.2 \mathrm{GHz}$ with a step $\Delta f=22.2 \mathrm{MHz}$. Figure $2(\mathrm{~b})$ is the time domain B-scan raw data of the simulated received signal. Frequency domain data is obtained, by performing an FFT on the B-scan time domain signal, and is used in the imaging.

In the following simulations, unless otherwise specified, we assume that the wall dielectric constant is known and is used in the imaging. The estimation of wall parameters associated with single or multilayered building walls has been extensively studied in the past decade within the framework of a one-dimensional inverse scattering problem [27-34]. For a single-layer wall, radar-based closed-form estimation of the wall dielectric constant, conductivity, and thickness is given in $[30,31]$.

Figure 2(c) shows the imaging result of the steel bars inside the wall using the proposed algorithm with the known wall dielectric constant $\varepsilon_{b}=6$, in which the back wall is highlighted with a dashed black line. From this figure, we find that the 20 sinusoid distributed steel bars and the back 


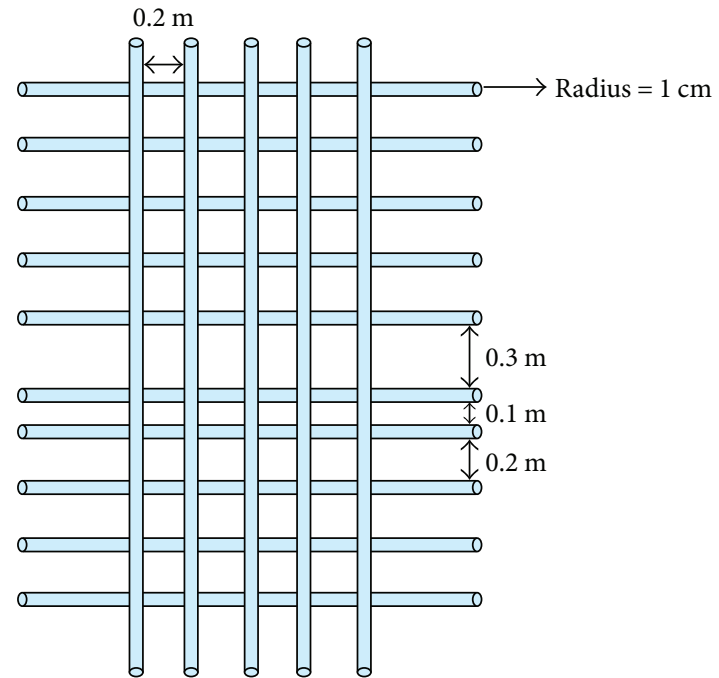

(a)

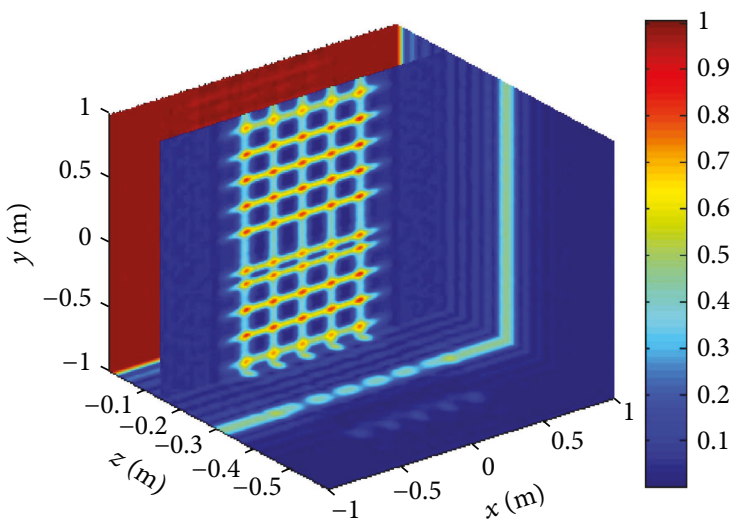

(b)

FIgURE 6: Imaging of the grid reinforcement. (a) Geometrical layout of the reinforcement grid inside the wall. (b) 3-D imaging result.

boundary of the wall are clearly identified and correctly localized at their true locations. For comparison, the imaging results using the ray tracking-based back-projection algorithm from [19] are provided in Figure 2(d). It takes about $57.34 \mathrm{~s}$ to reconstruct the image using the ray tracing-based back-projection algorithm. The proposed imaging algorithm, however, requires only $0.64 \mathrm{~s}$ to reconstruct the image in Figure 2(c) on the same computer. Due to the utilization of FFT/IFFT in the proposed imaging algorithm, the imaging process can be achieved in a short computation time, which is desirable for on-site applications. From the comparison of Figures 2(c) and 2(d), we also find less cluttered and a cleaner image can be achieved using the proposed algorithm due to its more accurate modeling of the wave propagation phenomenon through incorporation of the halfspace Green's function.

Incorrect estimation of the wall parameters could result in the shift of target position and defocussing of the target image. Figures 2(e) and 2(f) show the imaging result with an underestimated wall parameter $\left(\varepsilon_{b}=3\right)$ and an overestimated wall parameter $\left(\varepsilon_{b}=10\right)$. In the two figures, the back boundary of the wall is highlighted with a dashed black line. It is observed from the two figures that underestimation of the wall dielectric constant causes a shift of the target position toward the radar in the downrange. Overestimation of the wall dielectric constant causes a shift of targets position away from the radar system in downrange.

In the above simulation, the radar scans the wall from $-2 \mathrm{~m}$ to $2 \mathrm{~m}$ at a step size of $2.5 \mathrm{~cm}$. To investigate the effect of step size on the imaging, we present imaging results with $2.5 \mathrm{~cm}, 5 \mathrm{~cm}$, and $10 \mathrm{~cm}$ step sizes in Figures $3(\mathrm{a})-3(\mathrm{c})$, respectively. From the three figures, we find degradation in the azimuth resolution by increasing the step size of the synthesized aperture. The minimum step size should be no less than the Nyquist sampling interval $\lambda / 2 \sqrt{\varepsilon_{b}}$, where $\lambda$ is the wavelength of the transmitted signal and $\varepsilon_{b}$ is the dielectric constant of the wall.
The downrange resolution is primarily determined by the bandwidth of the transmitted signal. Figures $4(\mathrm{a})-4(\mathrm{c})$ are the imaging results with $2.4 \mathrm{GHz}, 1.2 \mathrm{GHz}$, and $0.6 \mathrm{GHz}$ bandwidth, respectively. As expected, the downrange resolution degrades as the bandwidth is reduced. At the narrowest bandwidth of $0.6 \mathrm{GHz}$, we can only resolve the front and back boundaries of the wall but cannot resolve the steel bars inside the wall, as shown in Figure 4(c).

To quantitatively characterize the results, the signal fidelity factor in [35-37] is calculated to quantify the similarity of the received and transmitted signals. In order to compare only the shape of the signals but not their magnitudes, the received and the transmitted signals are normalized according to

$$
\begin{aligned}
& \widehat{R}_{S}(t)=\frac{R_{S}(t)}{\left[\int_{-\infty}^{\infty} R_{S}^{2}(t) d t\right]^{1 / 2}}, \\
& \widehat{T}_{S}(t)=\frac{T_{S}(t)}{\left[\int_{-\infty}^{\infty} T_{S}^{2}(t) d t\right]^{1 / 2}} .
\end{aligned}
$$

The signal fidelity factor (SFF) is then defined as the peak of the cross-correlation of the normalized transmitted and received signals and is given as [37]

$$
\mathrm{SFF}=-\min _{n} \int_{-\infty}^{\infty} \widehat{T}_{S}(t) \widehat{R}_{S}(t+\tau) d t
$$

We note that a 180-degree phase change occurs as the signal is reflected from the wall; thus, the minimum of the correlation is used and a minus sign is included in (22). Figure 5 shows the SFF of the reinforcement wall, shown in Figure 2(a), at different antenna locations. For a perfect metal wall, the SFF is expected to be 1 as no distortion occurs in the shape of the transmitted and received signal. Due to the scattering from the steel bars and the multiple scattering effects inside the wall, signal distortion occurs as it is reflected from a reinforced wall. 


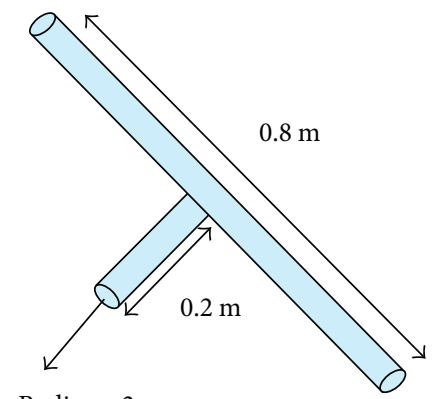

Radius $=2 \mathrm{~cm}$

(a)

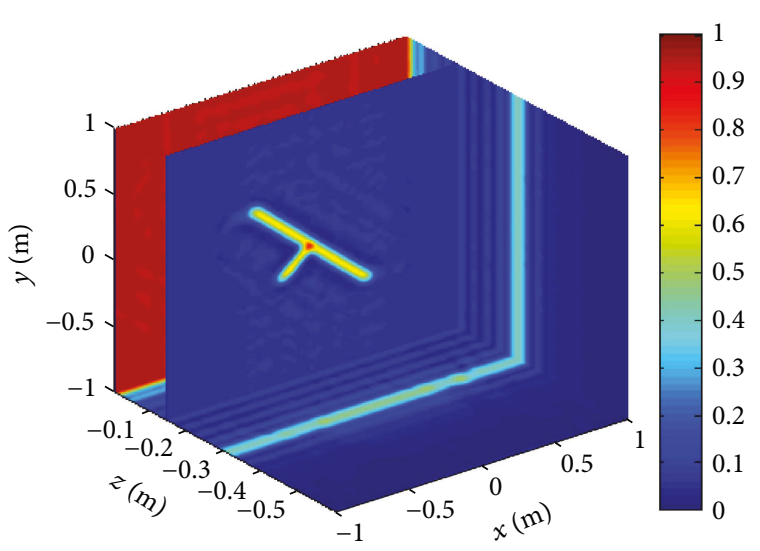

(b)

Figure 7: 3-D imaging of the tubes hidden inside the wall. (a) Dimension of tubes and (b) 3-D imaging result.

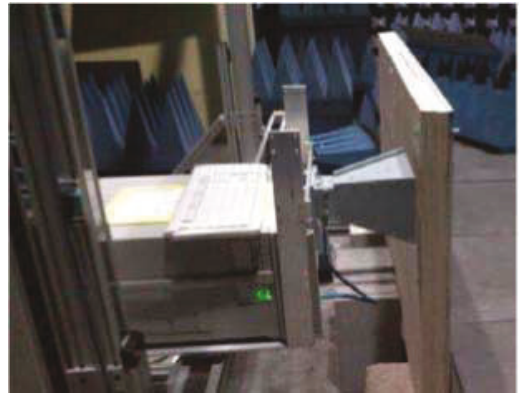

(a)

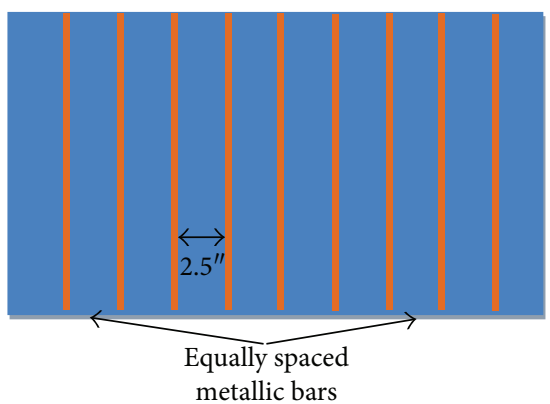

(b)

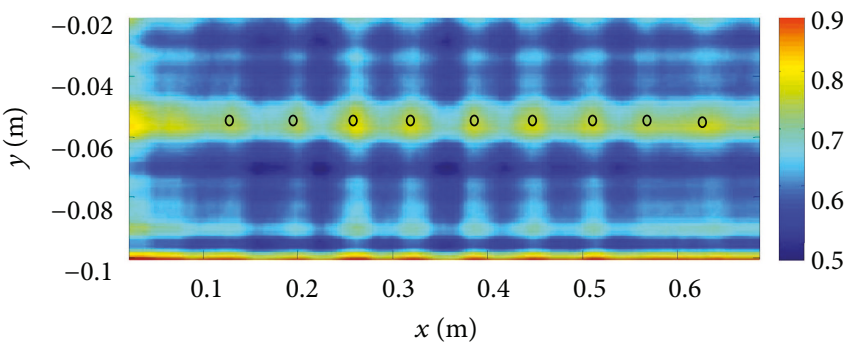

(c)

FiguRE 8: 1/3 scale cement board with metallic inclusions. (a) Measurement setup. (b) Layout of the wall interior. (c) Imaging result. The small circles indicate the true locations of the metallic bars.

In the second example, we present the 3-D imaging of a reinforcement grid inside the wall. Figure 6(a) shows the dimensions of the reinforcement grid and the interelement spacing between the reinforcement bars. The radius of each steel bar is $1 \mathrm{~cm}$. The spacing between the fifth and sixth reinforcement bars is $0.3 \mathrm{~m}$, and the spacing between the sixth and seventh reinforcement bars is $0.1 \mathrm{~m}$ in the vertical direction. The interelement spacing between other steel bars is $0.2 \mathrm{~m}$. The permittivity, conductivity, and thickness of the wall are $\varepsilon_{b}=6, \sigma=0.01 \mathrm{~S} / \mathrm{m}$, and $d=0.3 \mathrm{~m}$, respectively. The simulation was performed over a $2 \mathrm{~m} \times 2 \mathrm{~m}$ square aperture parallel to the wall at a standoff distance of $0.1 \mathrm{~m}$. The operating frequency covers a range from $0.8 \mathrm{GHz}$ to $2.5 \mathrm{GHz}$ with a step of $26 \mathrm{MHz}$. The imaging region is
$2 \mathrm{~m} \times 2 \mathrm{~m} \times 0.6 \mathrm{~m}$ and is discretized into $160 \times 160 \times 80$ pixels. Figure 6(b) shows the 3-D imaging result of the reinforcement grid inside the wall. From this figure, it is clear that the proposed algorithm can successfully identify and correctly localize not only the back boundary of the wall but also all the steel bars inside the wall. The large spacing between the fifth and the sixth steel bars and the small spacing between the sixth and seventh steel bars can be clearly observed in the image. The 3-D imaging of the reinforcement grid in Figure 6(b) took only $28.6 \mathrm{~s}$ using the proposed imaging algorithm.

In the last example, we present the 3-D imaging of two perpendicular tubes hidden inside the wall. The dimension and orientations of the tubes are shown in Figure 7(a). The lengths of the long and short tubes are $0.8 \mathrm{~m}$ and $0.2 \mathrm{~m}$, 


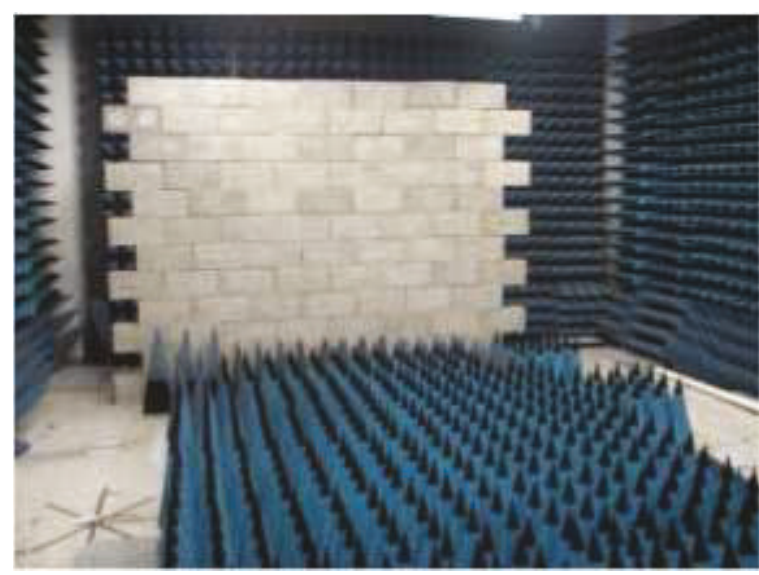

(a)

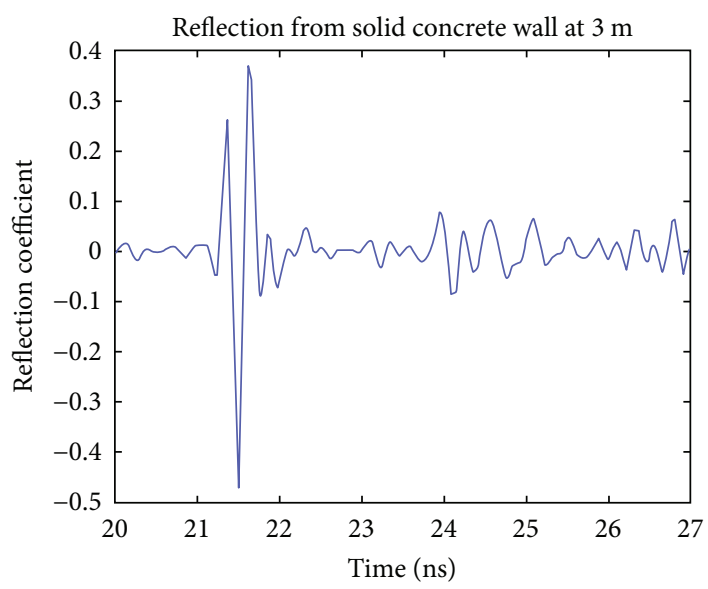

(c)

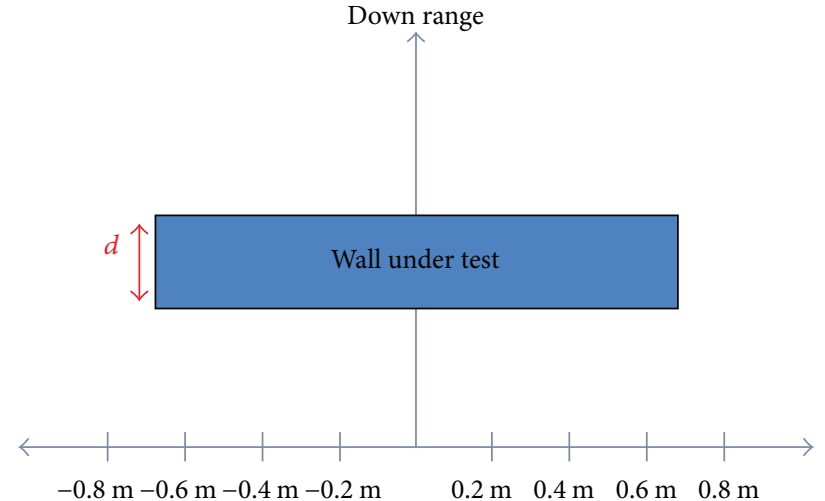

Cross range

(b)

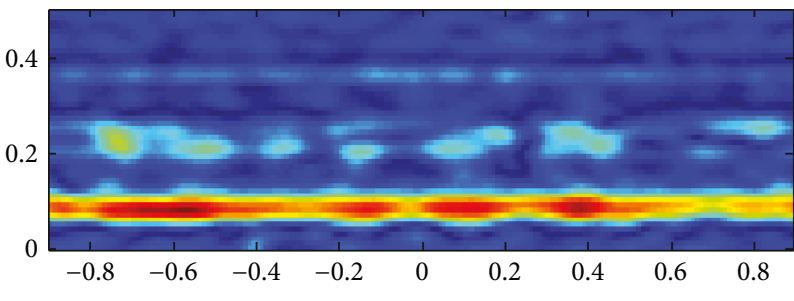

(d)

FIGURE 9: Solid concrete block wall with no backing. (a) Measurement setup. (b) Geometry. (c) Recorded reflection coefficient. (d) Imaging result.

respectively. Figure 7 (b) shows the 3-D imaging result of the two tubes inside wall. We find that the long tube and short tube can be clearly observed in the 3-D image and the orientations of the tubes are also clearly identified. The successful retrieval of the width, length, and orientation of the concealed objects inside the wall provides valuable information for building maintenance and building quality and durability assessment as well as in detection of weapons and other related objects in various homeland security and lawenforcement scenarios. It took only $28.3 \mathrm{~s}$ to reconstruct the 3-D image. The fast imaging speed shows the promise of the proposed 3-D intrawall imaging for on-site applications.

\section{Experimental Results}

Some experimental examples are presented in this section to verify the proposed algorithm for $2-\mathrm{D}$ and $3-\mathrm{D}$ intrawall imaging in a controlled lab environment. The 2-D imaging algorithm was applied to three experimental cases of a solid concrete wall with (1) embedded rebar, (2) the wall with no backing, and (3) a mirror placed on the back face of the wall. The last test of the mirror behind the concrete wall was also processed using the 3-D imaging algorithm.

The 2-D intrawall imaging algorithm was applied to the case of metallic rebar embedded within a solid concrete wall. Due to the limitations of fabricating this type of scenario in full scale, a $1 / 3$ scale model was created which utilized multiple layers of a cement board readily available in home improvement stores. Multiple layers of the board were required as it was sourced at $1 / 4^{\prime \prime}$ thickness, and thus to make a 1/3 scale replica of a full thickness wall, multiple pieces were layered to create a single wall. This layering process allowed for the inclusion of metallic strips which were analogous to metallic rebar placed within a full size solid concrete wall. Additionally, because of the layering, the metallic strips were composed of copper tape in 3-layer thickness. Thicker strips could not be used while still maintaining a continuous contact between cement board layers. Due to the $1 / 3$ scale of the wall, the frequency range for the test was increased by a factor of 3 to cover $3 \mathrm{GHz}$ to $9 \mathrm{GHz}$ which corresponds to a 


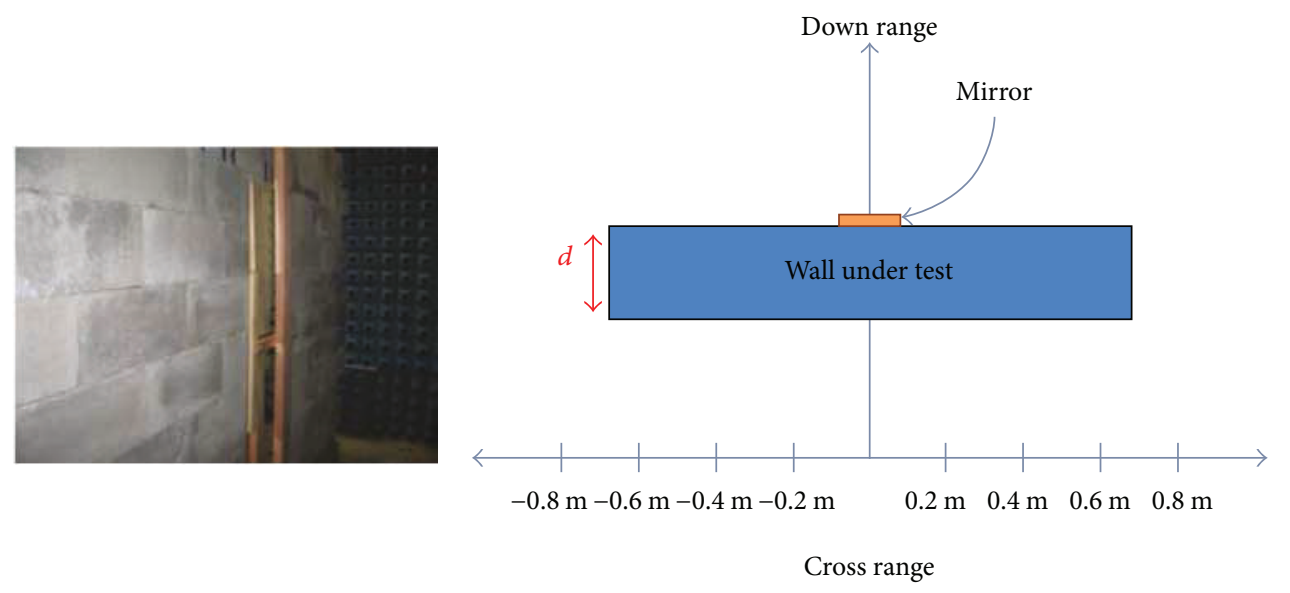

(a)

(b)

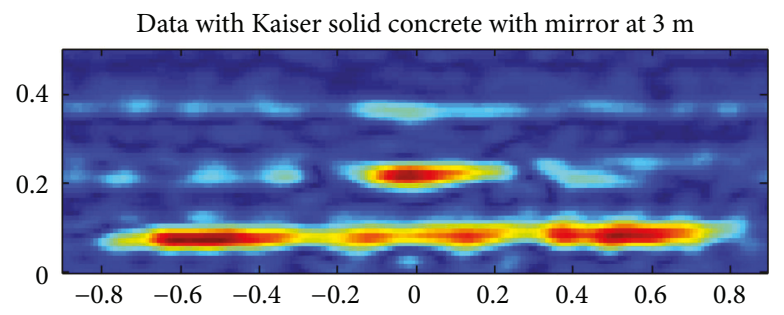

(c)

Figure 10: (a) Photo from behind the solid concrete block wall with centered vertical mirror in direct wall contact. (b) Measurement setup and geometry. (c) Imaging result using the proposed 2-D microwave tomographic algorithm.

standard TWRI frequency range of $1 \mathrm{GHz}$ to $3 \mathrm{GHz}$ without scaling. An Agilent PNA with an associated horn antenna which covered $3-9 \mathrm{GHz}$ was used to measure the wall analogue. The measurement setup along with the diagram of the wall with metallic strip locations is shown in Figure 8. The resulting image using the proposed 2-D imaging algorithm is shown in Figure 8 where the metallic inclusions are well localized within the center of the wall. Additionally, striations are visible in the image corresponding to the interfaces between the various layers of cement board.

In the next set of experiments, radar measurements were performed for a concrete wall, with and without backing. The wall under test was constructed using solid concrete bricks with a known dielectric constant of 7.66 and a loss tangent of $0.158 \mathrm{~S} / \mathrm{m}$ and downrange thickness of 6 inches. The measurement array was fixed at $3 \mathrm{~m}$ standoff distance, measured from the front of the wall to the feed point on the antenna. The backing, which was a mirror, was placed at the rear surface of the wall in a configuration which allowed for the most direct contact between the backing and the rear surface of the wall. A frequency range of $0.7 \mathrm{GHz}$ to $3.1 \mathrm{GHz}$ was chosen for imaging using a step size of $3 \mathrm{MHz}$. An Agilent ENA 5071B was used for signal transmission and data collection. A dual-polarized horn antenna, with an operational bandwidth from $0.7 \mathrm{GHz}$ to $6 \mathrm{GHz}$, was used as the transceiver. Figure 9 shows the experimental setup of the solid concrete wall with no backing, a selected reflection signal from the wall, and its associated image resulting from the proposed 2-D microwave tomographic technique. Figure 10 shows the results of

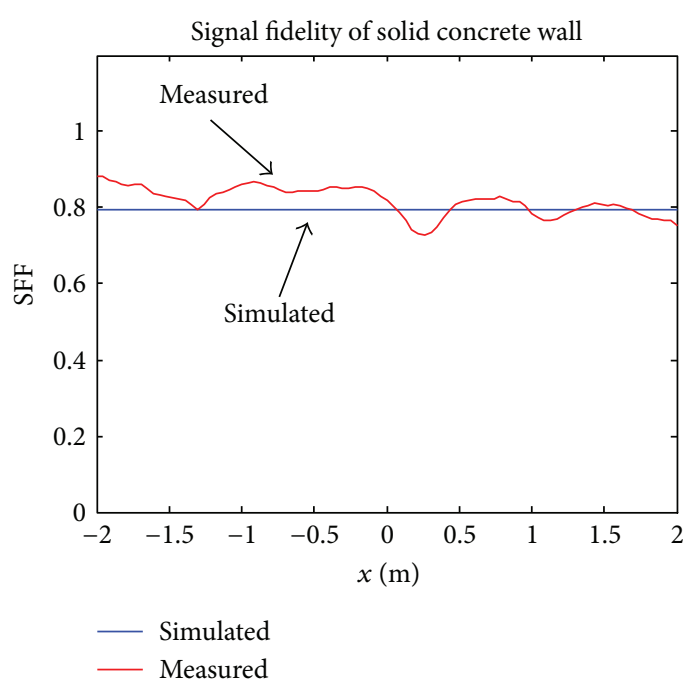

FIGURE 11: SFF of the received radar signals from the solid concrete wall.

imaging the same solid concrete wall of Figure 9 with the addition of a vertically oriented mirror placed directly behind the wall and in direct contact with the wall. In Figure 10(c), the imaging result clearly shows the reflections from the front of the wall, at $0.05 \mathrm{~m}$, and the mirror on the back of the wall, at $0.2 \mathrm{~m}$. The reflection from the rear face of the wall is low compared to that of the mirror but is still visible at $0.2 \mathrm{~m}$ downrange to the left and right of the mirror reflection. 


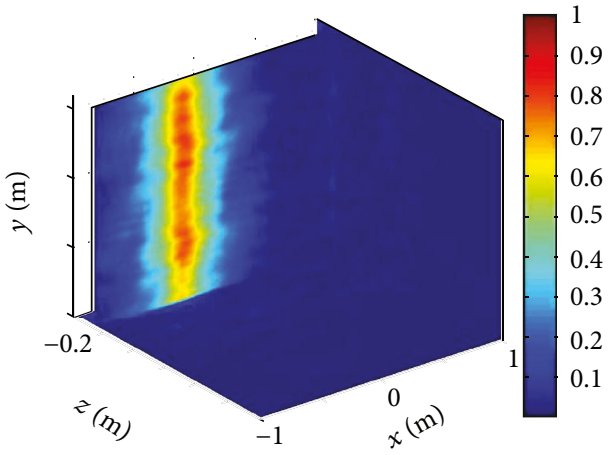

(a)

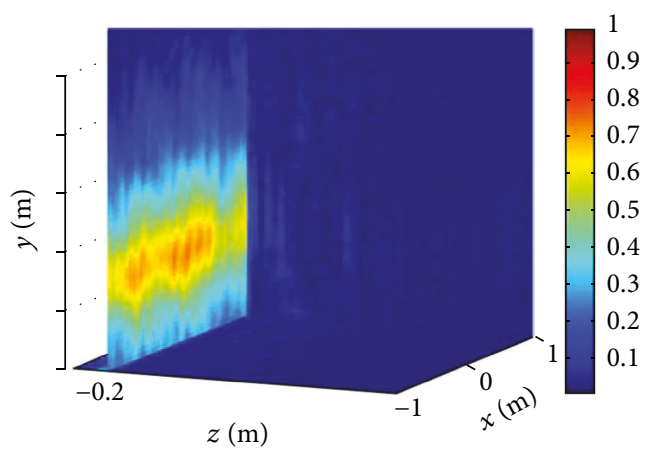

(b)

FIGURE 12: Imaging results using the proposed 3-D microwave tomographic algorithm for (a) vertically and (b) horizontally oriented mirror behind a solid concrete wall.

The third ringing of the wall and mirror can also be seen in this image at $0.37 \mathrm{~m}$. In all cases, the computation time was less than 1 second for image generation. In Figure 11, the comparison of the SFF calculations for the solid concrete wall using simulated and measured results is presented. A relatively close agreement between the SFF values of simulated and measured received signals versus the antenna positions is obtained.

The 3-D imaging algorithm, discussed above, was also applied to the measured scenario of a mirror on the rear surface of the wall in both vertical and horizontal configurations. Measured data was collected in a grid with 97 positions in the $\mathrm{x}$-direction and 23 positions in the $\mathrm{y}$-direction and at a $3 \mathrm{~m}$ standoff distance from the wall. At each location, step frequency data was collected from $1 \mathrm{GHz}$ to $6 \mathrm{GHz}$ with 1601 frequency steps. Both measured data sets were then processed using the 3-D microwave tomography algorithm given in (15). Figures 12(a) and 12(b) show a side by side comparison of the vertical and horizontal mirror imaging results. In both cases, the mirror orientation is readily identifiable. We note that the above and other cases of detection and imaging of the wall's conductor backings are of interest in the planning of various search and rescue mission scenarios. Additionally, it is of note that the experimental results shown in Figures 8-11 are individual experiments which are representative examples from the multiple data collections performed during this research. These results are not an average over the many experiments performed but rather are selected examples which are indicative of the results.

\section{Conclusion}

The fast and nondestructive inspection of wall interior structures has promising applications in building safety and durability assessment as well as in various defense and lawenforcement scenarios. In this paper, a fast and efficient microwave tomographic algorithm is proposed for near real-time 2-D and 3-D intrawall imaging. The linearization of the inversion scheme and employment of the FFT/IFFT in the imaging formulation make the proposed imaging algorithm suitable for various applications pertaining to the inspection of a large probed region and allow real-time processing. Numerical and experimental 2-D and 3-D examples are presented with each showing the successful retrieval and localization of the wall interior or wall backing information using the proposed imaging algorithm. It takes less than one second/minute for 2-D/3-D imaging using the proposed algorithm, a feature that is very attractive for many applications which require on-site processing. Although this paper is concentrated on the intra-wall inspection, the presented algorithm is applicable to other concrete structure nondestructive testing, such as bridge/dam defect characterization, pavement inspection, and archeology. We note that the proposed radar imaging algorithm utilizes a linearization scheme to significantly speed up the imaging of intrawall objects and results in the object's accurate geolocation and shape reconstruction but not its material composition, such as permittivity and loss tangent. The later requires nonlinear inverse-profiling schemes which usually necessitate a numerically computed forward model and a global optimization inversion scheme thus making the inversion very timeconsuming and not suitable for on-site processing.

\section{Conflicts of Interest}

The authors declare that they have no conflicts of interest.

\section{References}

[1] V. Barrilea and R. Pucinotti, "Application of radar technology to reinforced concrete structures: a case study," NDT \& E International, vol. 38, no. 7, pp. 596-604, 2005.

[2] A. Boryssenko, O. Boryssenko, A. Lishchenko, and V. Prokhorenko, "Inspection of internal structure of walls by subsurface radar," IEEE Aerospace and Electronic Systems Magazine, vol. 21, no. 10, pp. 28-31, 2006.

[3] J. Bungey, "Sub-surface radar testing of concrete: a review," Construction and Building Materials, vol. 18, no. 1, pp. 1-8, 2004.

[4] D. M. McCann and M. C. Forde, "Review of NDT methods in the assessment of concrete and masonry structures," NDT \& $E$ International, vol. 34, no. 2, pp. 71-84, 2001. 
[5] F. Ahmad, Y. Zhang, and M. Amin, "Three-dimensional wideband beamforming for imaging through a single wall," IEEE Geoscience Remote Sensing Letters, vol. 5, no. 2, pp. 176179, 2008.

[6] W. Zhang and A. Hoorfar, "A generalized approach for SAR and MIMO radar imaging of building interior targets with compressive sensing," IEEE Antennas and Wireless Propagation Letters, vol. 14, pp. 1052-1055, 2015.

[7] E. J. Baranoski, "Through wall imaging: historical perspective and future directions," in 2008 IEEE International Conference on Acoustics, Speech and Signal Processing, pp. 5173-5176, Las Vegas, NV, USA, 2008.

[8] M. G. Amin, F. Ahmad, and W. Zhang, "Target RCS exploitations in compressive sensing for through-the-wall radar imaging," in 2010 International Waveform Diversity \& Design Conference, pp. 150-154, Ontario, Canada, 2010.

[9] J. D. Zaeytijd, T. Maes, and A. Franchois, "2-D real-time quantitative microwave imaging of reinforcement bars: simulations and experiments," in 2007 International Conference on Electromagnetics in Advanced Applications, pp. 145-148, Torino, Italy, 2007.

[10] K. J. Langenberg, M. Brandfaß, R. Hannemann et al., "Inverse scattering with acoustic, electromagnetic and elastic waves as applied in nondestructive evaluation," in Wavefield Inversion. International Centre for Mechanical Sciences (Courses and Lectures), A. Wirgin, Ed., vol. 398, Springer, Vienna, 1999.

[11] W. Zhang, "Three-dimensional through-the-wall imaging with MIMO radar," Journal of Electromagnetic Waves and Applications, vol. 28, no. 15, pp. 1935-1943, 2014.

[12] T. J. Cui and W. C. Chew, "Diffraction tomographic algorithm for the detection of three-dimensional objects buried in a lossy half space," IEEE Transactions on Antennas and Propagation, vol. 50, no. 1, pp. 42-49, 2002.

[13] R. Deming and A. J. Devaney, "Diffraction tomography for multi-monostatic ground penetrating radar imaging," Inverse Problems, vol. 13, no. 1, pp. 29-45, 1997.

[14] A. Brancaccio, R. Solimene, G. Prisco, G. Leone, and R. Pierri, "Intra-wall diagnostics via a microwave tomographic approach," Journal of Geophysics and Engineering, vol. 8, no. 3, pp. S47-S53, 2011.

[15] G. Bozza, M. Brignone, M. Pastorino, M. Piana, and A. Randazzo, "Crack detection in dielectric structures by a linear sampling approach," International Journal of Signal and Imaging Systems Engineering, vol. 3, no. 2, pp. 73-80, 2010.

[16] I. Catapano and L. Crocco, "An imaging tool for intra-wall investigations: a feasibility study," in Proceedings of the XXIXth URSI General Assembly in Chicago, 2008.

[17] X. Chen, P. Li, and S. P. Yeo, "Multiple signal classification algorithm for non-destructive imaging of reinforcement bars and empty ducts in circular concrete columns," in 2011 XXXth URSI General Assembly and Scientific Symposium, Istanbul, Turkey, 2011.

[18] Y. J. Kim, L. Jofre, F. D. Flaviis, and M. Q. Feng, "Microwave reflection tomographic array for damage detection of civil structures," IEEE Transactions on Antennas and Propagation, vol. 51, no. 11, pp. 3022-3032, 2003.

[19] T. Counts, A. C. Gurbuz, W. R. Scott, J. H. McClellan, and K. Kim, "Multistatic ground-penetrating radar experiments," IEEE Transactions on Geoscience and Remote Sensing, vol. 45, no. 8, pp. 2544-2553, 2007.
[20] Y. J. Kim, L. Jofre, F. D. Flaviis, and M. Q. Feng, "Threedimensional microwave imaging technology for damage detection of concrete structures," in Proceedings Volume 5057, Smart Structures and Materials 2003: Smart Systems and Nondestructive Evaluation for Civil Infrastructures, San Diego, California, USA, 2003.

[21] J. Hugenschmidt and A. Kalogeropoulos, "The inspection of retaining walls using GPR," Journal of Applied Geophysics, vol. 67, no. 4, pp. 335-344, 2009.

[22] L. Tsang, J. A. Kong, and K. Ding, Scattering of Electromagnetic Waves, Theories and Applications, John Wiley \& Sons, Inc, 2000.

[23] W. C. Chew, Waves and Fields in Inhomogeneous Media, IEEE Press, Piscataway, NJ, 1997.

[24] W. Zhang, "Two-dimensional microwave tomographic algorithm for radar imaging through multilayered media," Progress In Electromagnetics Research, vol. 144, pp. 261-270, 2014.

[25] M. Chiappe and G. L. Gragnani, "An analytical approach to the reconstruction of the radiating current in inverse electromagnetic scattering," Microwave and Optical Technology Letters, vol. 49, no. 2, pp. 354-360, 2007.

[26] C. J. Leuschen and R. G. Plumb, “A matched-filter-based reverse-time migration algorithm for ground-penetrating radar data," IEEE Transactions on Geoscience and Remote Sensing, vol. 39, no. 5, pp. 929-936, 2001.

[27] O. Aly and A. S. Omar, "Reconstructing stratified permittivity profiles using super-resolution techniques," IEEE Transactions on Microwave Theory and Techniques, vol. 54, no. 1, pp. 492498, 2006.

[28] J. Zhang, M. Nakhkash, and Y. Huang, "Electromagnetic imaging of layered building materials," Measurement Science and Technology, vol. 12, no. 8, pp. 1147-1152, 2001.

[29] C. Thajudden, W. Zhang, and A. Hoorfar, "Theory and experiments on imaging of walls' interior structures using diffraction tomography," in Proceedings of the 2012 IEEE International Symposium on Antennas and Propagation, Chicago, IL, USA, July 2012.

[30] C. Thajudeen, A. Hoorfar, and W. Zhang, "Estimation of frequency-dependent wall parameters of unknown walls for enhanced through-the-wall imaging," in 2011 IEEE International Symposium on Antennas and Propagation (APSURSI), pp. 3070-3073, Spokane, WA, USA, 2011.

[31] M. Aftanas and M. Drutarovsky, "Imaging of the building contours with through the wall UWB radar system," Radio Engineering, vol. 18, pp. 258-264, 2009.

[32] C. Thajudeen and A. Hoorfar, "A hybrid bistatic-monostatic radar technique for estimation of lossy wall parameters," IEEE Antennas and Wireless Propagation Letters, vol. 16, pp. 1249$1252,2016$.

[33] M. Donelli, D. Franceschini, P. Rocca, and A. Massa, “Threedimensional microwave imaging problems solved through an efficient multiscaling particle swarm optimization," IEEE Transactions on Geoscience and Remote Sensing, vol. 47, no. 5, pp. 1467-1481, 2009.

[34] M. Hajebi, A. Hoorfar, E. BouDaher, and A. Tavakoli, "Inverse profiling of inhomogeneous subsurface targets with arbitrary cross sections using covariance matrix adaptation evolution strategy," IEEE Geoscience and Remote Sensing Letters, vol. 14, no. 5, pp. 612-616, 2017.

[35] D. H. Kwon, "Effect of antenna gain and group delay variations on pulse-preserving capabilities of ultrawideband 
antennas," IEEE Transactions on Antennas and Propagation, vol. 54, no. 8, pp. 2208-2215, 2006.

[36] E. Pancera, Strategies for Time Domain Characterization of UWB Components and Systems, 2009.

[37] G. Quintero, J. F. Zürcher, and A. K. Skrivervik, "System fidelity factor: a new method for comparing UWB antennas," IEEE Transactions on Antennas and Propagation, vol. 59, no. 7, pp. 2502-2512, 2011. 


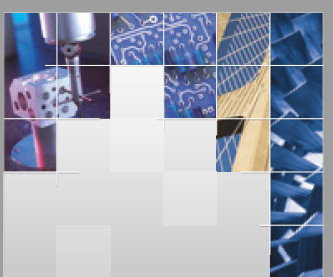

\section{Enfincering}
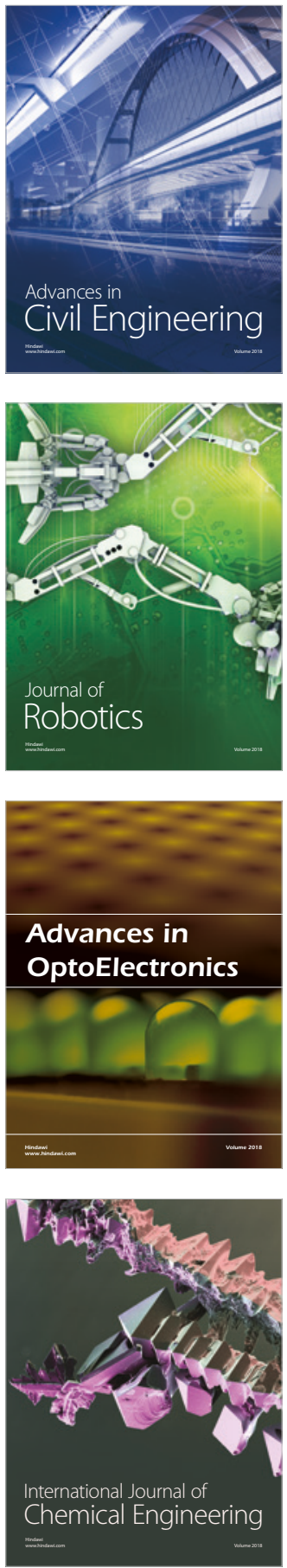

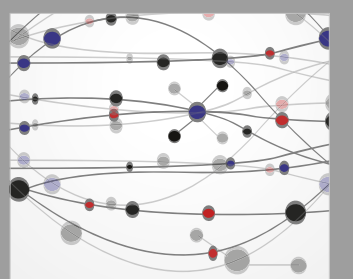

\section{Rotating \\ Machinery}

The Scientific World Journal

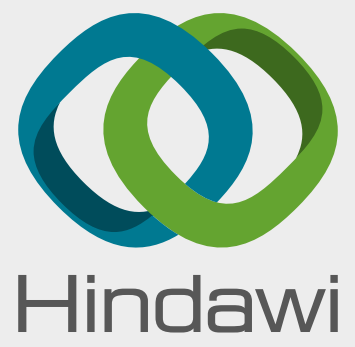

Submit your manuscripts at

www.hindawi.com
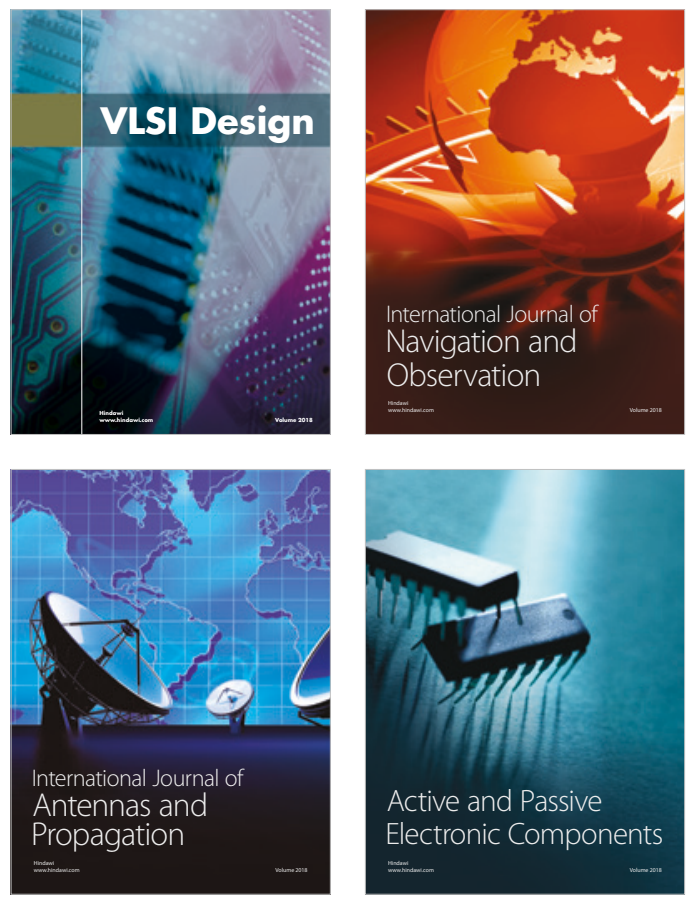
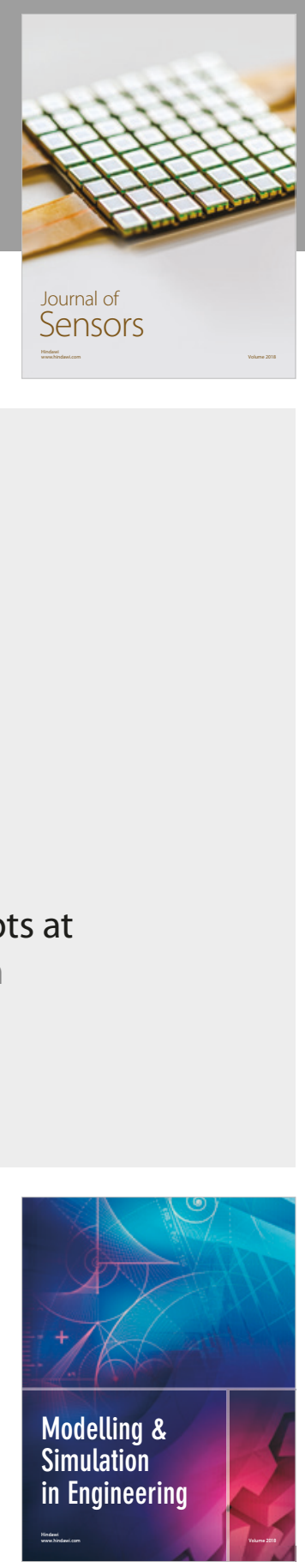

\section{Advances \\ Multimedia}
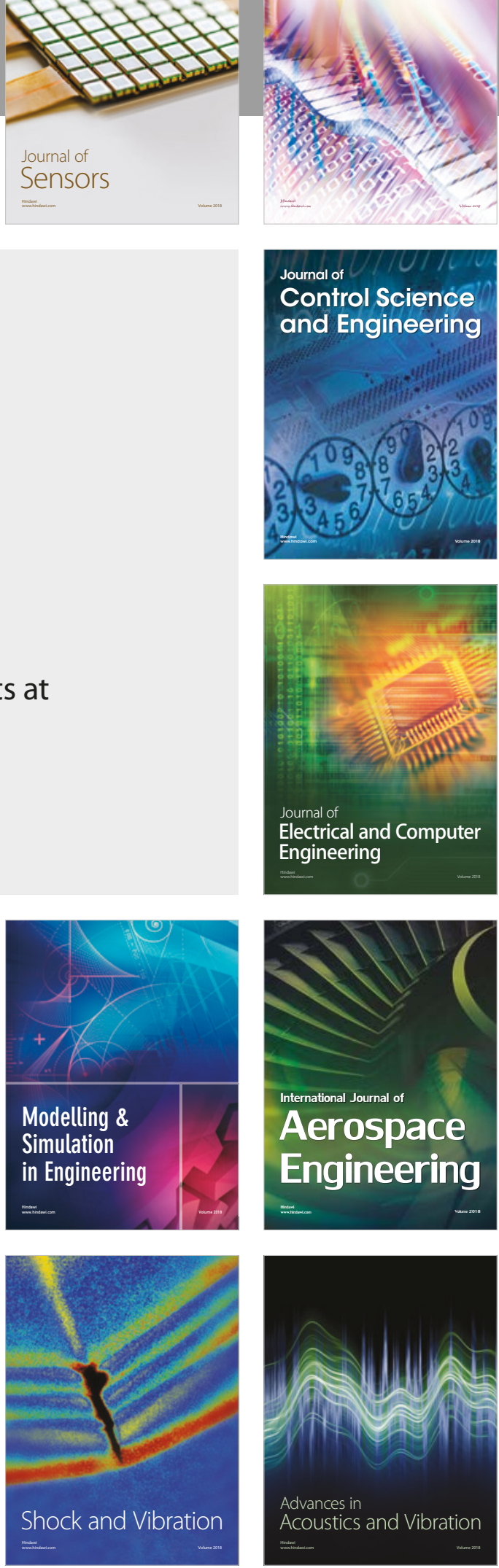